\title{
Molecular detection and pathology of Pasteurella multocida B:2 in the reproductive system of pre-pubertal buffalo calves (Bubalus bubalis)
}

\begin{abstract}
Pasteurella multocida is responsible for one of the major animal diseases with economic importance in both developed and developing countries. P. multocida B:2 causes haemorrhagic septicemia (HS) in cattle and buffaloes, which result in heavy economic losses through direct effect, resulting in high mortality rates and indirect effects through impairment of the animal productivity. It is well known that HS affects mainly the respiratory and digestive tracts of host animals. However, involvement of the reproductive system in the pathogenesis of HS has not been reported previously. This study was designed to present detailed detection and histopathological changes of the reproductive system and mammary glands of buffaloes experimentally infected with P. multocida B:2. Briefly, nine healthy buffalo calves aged 8-month-old were divided into three groups of three calves each. Calves from groups 1 and 2 were inoculated subcutaneously and orally with $10 \mathrm{~mL}$ of 1 政 cfu/mL of P. multocida B:2, respectively, while calves of group 3 were inoculated orally with $10 \mathrm{~mL}$ of phosphate buffer saline as a sham control. All the calves in group 1 had to be euthanized after $12 \mathrm{~h}$ post-infection, while calves in group 2 were euthanized after day 21 post-infection. Successful isolation and PCR confirmation of P. multocida B:2 was achieved from different parts of the reproductive system, including ovary, oviduct, uterine horn, uterine body and vagina as well as mammary glands and supramammary lymph nodes of the buffaloes in group 1 after $12 \mathrm{~h}$ post-infection. However, P. multocida B:2 was not detected in the reproductive organs of buffaloes in group 2 and group 3 after 21 days post-infection. Significant differences (P\&llo5) were found in histopathological changes between the two groups, which mainly affected different anatomic regions of the reproductive system. This work provides an insight into the involvement of the female reproductive system of buffaloes during the pathogenesis of HS and shows that route of inoculation strongly affect the localization of the bacterium in the reproductive system.
\end{abstract}

Keyword: Buffaloes; Female reproductive system; Histopathology; Mammary gland; Pasteurella multocida B:2; PCR 